\title{
Treatment of a GIST patient with modified dose of sunitinib by measurement of plasma drug concentrations
}

\author{
TADAHIRO HASHITA ${ }^{1,3}$, YOSHIHIKO KATSUYAMA ${ }^{1}$, KATSUNORI NAKAMURA $^{1}$, \\ YASUYUKI MOMOSE ${ }^{1}$, DAISUKE KOMATSU ${ }^{2}$, NAOHIKO KOIDE ${ }^{2}$, SHINICHI MIYAGAWA ${ }^{2}$, \\ TOMONORI NAKAMURA $^{3}$, KOUJIROU YAMAMOTO $^{3}$ and SHIGERU OHMORI $^{1}$ \\ Departments of ${ }^{1}$ Pharmacy and ${ }^{2}$ Surgery, Shinshu University Hospital, Matsumoto; \\ ${ }^{3}$ Department of Clinical Pharmacology, Gunma University Graduate School of Medicine, Maebashi, Japan
}

Received March 1, 2012; Accepted June 12, 2012

DOI: $10.3892 / \mathrm{ol} .2012 .779$

\begin{abstract}
In this study we report the pharmacokinetics and severe adverse effects of sunitinib in a woman with a gastrointestinal stromal tumor (GIST). A 60-year-old woman with small intestinal GIST developed severe thrombocytopenia $\left(1.7 \times 10^{4} / \mu 1\right)$ following 1 week of treatment with sunitinib at $50 \mathrm{mg} /$ day. Although the dose of sunitinib was reduced to $25 \mathrm{mg} /$ day, platelet levels remained low. On day 7, the trough concentration of sunitinib plus SU12662 was $46.1 \mathrm{ng} / \mathrm{ml}$ and the area under the curve (AUC) was 1,393.0 $\mathrm{ng} \cdot \mathrm{h} / \mathrm{l}$. The dose was again reduced to $12.5 \mathrm{mg} /$ day. However, the day after resumption of treatment, the patient developed symptoms of left heart failure due to myocardosis caused by sunitinib. Sunitinib has been reported to inhibit platelet-derived growth factor receptor (PDGFR) phosphorylation at concentrations over the range of $50-100 \mathrm{ng} / \mathrm{ml}$ (sunitinib plus SU12662) in vivo. In this case, the plasma concentration was sufficient to inhibit PDGFR at 25 or $50 \mathrm{mg}$ /day. However, thrombocytopenia appeared at both dosages. Although the results in this case did not suggest a correlation between thrombocytopenia and plasma concentration, the degree of thrombocytopenia was decreased by reduction of the dose. In conclusion, the findings reported here indicate that the plasma concentration of sunitinib plus SU12662 is an important indicator to reduce adverse effects.
\end{abstract}

Correspondence to: Professor Shigeru Ohmori, Department of Pharmacy, Shinshu University Hospital, 3-1-1 Asahi, Matsumoto 390-8621, Japan

E-mail: somori@shinshu-u.ac.jp

Abbreviations: GIST, gastrointestinal stromal tumor; KIT, stem cell factor receptor; Flk-1/KDR, fetal liver kinase-1/kinase-insert domain-containing receptor; PDGFR, platelet-derived growth factor receptor- $\alpha$; VEGFR, vascular endothelial growth factor receptor; FLT3, FMS-like tyrosine kinase 3; CSF-1, colony-stimulating factor 1; RET, glial cell line-derived neurotrophic factor receptor (rearranged during transfection); CYP, cytochrome P450; AUC, area under curve

Key words: sunitinib, SU12662 (N-desethyl sunitinib), gastrointestinal stromal tumor, thrombocytopenia

\section{Introduction}

Most gastrointestinal stromal tumors (GIST) have activating mutations in stem cell factor receptor (KIT) or platelet-derived growth factor receptor- $\alpha$ (PDGFRA) (1). Approximately 85\% of GIST cases have activating mutations in KIT, and 5\% have activating mutations in PDGFRA (1). Although the KIT gene contains 21 exons, KIT in GIST only has mutations within exons $8,9,11,13,14,17$ and 18 . Most mutations (70\%) in the KIT gene have been reported in the juxtamembrane domain (exon 11), and $15 \%$ of cases have mutations in the extracellular domain (exon 9) (2).

Imatinib mesylate (Glivec ${ }^{\circledR}$; Novartis Pharma, Basel, Switzerland) selectively inhibits KIT and PDGFRA and is the first-line treatment in adult patients with KIT-positive non-resectable malignant GIST. The standard dose of imatinib is $400 \mathrm{mg}$ daily. Recently, it was reported that a high dose of imatinib (800 mg daily) resulted in a significantly superior progression-free survival rate in patients with GIST harboring a $K I T$ gene exon 9 mutation (3). It was suggested that KIT exon 9 mutation caused resistance to imatinib.

Sunitinib malate (Sutent $^{\circledR}$; Pfizer, New York, NY, USA) is a multi-target tyrosine kinase inhibitor and the second-line treatment of GIST following disease progression in cases intolerant to imatinib. Sunitinib inhibits KIT, PDGFRA, PDGFRB, vascular endothelial growth factor receptor (VEGFR), FMS-like tyrosine kinase 3 (FLT3), colony-stimulating factor 1 (CSF-1) and glial cell line-derived neurotrophic factor receptor rearranged during transfection (RET) (4-5). Sunitinib is metabolized by cytochrome $\mathrm{P} 450$ (CYP) 3A4, and produces $N$-desethyl metabolite, SU12662. SU12662 is an active metabolite that inhibits KIT, PDGFR and VEGFR in a similar manner to sunitinib. Previous animal studies demonstrated that target plasma concentrations of sunitinib plus SU12662 for the inhibition of PDGFRB and fetal liver kinase-1/kinaseinsert domain-containing receptor (Flk-1/KDR)/VEGFR-2 phosphorylation were in the range of $50-100 \mathrm{ng} / \mathrm{ml}$ (4). In addition, it is more crucial to maintain the effective concentration $(50-100 \mathrm{ng} / \mathrm{ml})$ than to obtain a high maximum plasma concentration (Cmax).

The metabolic capability of CYP3A4 varies greatly among individuals (6). Although most medications have various 
metabolic pathways, the plasma concentration of sunitinib varies greatly among individuals as sunitinib is metabolized only by CYP3A4.

Here, we describe a patient who developed thrombocytopenia while taking sunitinib. We assayed the plasma concentrations of sunitinib and SU12662 to avoid thrombocytopenia.

\section{Patients and methods}

Case report. A 60-year-old Japanese woman took sunitinib $50 \mathrm{mg}$ once daily after breakfast. The patient's height and weight were $150 \mathrm{~cm}$ and $36.45 \mathrm{~kg}$, respectively, resulting in a body surface area of $1.25 \mathrm{~m}^{2}$. Her medical history consisted of small intestinal GIST, which was immunohistochemically positive for KIT, smooth muscle actin, CD34 and vimentin. The tumor metastasized to the liver. She then took imatinib $400 \mathrm{mg}$ once daily after breakfast for 3 years. The dose of imatinib was reduced to $300 \mathrm{mg}$ once daily due to the appearance of adverse effects. However, the dose of imatinib was increased to $400 \mathrm{mg}$ once daily due to liver metastasis.

She was admitted to Shinshu University Hospital for one week for the first administration of sunitinib since there was no decrease in liver metastases following the change to the higher dose of imatinib. Fourteen days after the first administration of sunitinib, the patient experienced nosebleeds, stomatitis and malaise. The platelet (PLT) count was decreased to $1.7 \times 10^{4} / \mu 1$, which was categorized as grade 4 thrombocytopenia according to the National Cancer Institute criteria version 4.0. Sunitinib was then discontinued and the patient was admitted to our hospital. The PLT count was increased following administration of PLT and adverse effects were eliminated (Fig. 1). The administration of sunitinib was resumed at $25 \mathrm{mg}$ once daily and continued for 3 weeks. However, sunitinib was discontinued as the PLT count again decreased.

The PLT count normalized approximately 9 days after the discontinuation of sunitinib. Sunitinib was resumed at a dose of $12.5 \mathrm{mg}$ once daily and the plasma concentrations of sunitinib and its metabolite, SU12662, were analyzed (Fig. 2). However, the patient developed a dry cough the day after resumption of sunitinib. A computed tomography scan revealed interstitial pneumonia. Echocardiography revealed hypokinesis of the left ventricle, which was shown to be drug-induced heart failure.

Compounds. Sutent $12.5 \mathrm{mg}$ was purchased from Pfizer Global Research and Development (Japan). Sunitinib malate and $N$-desethyl sunitinib (SU12662) were purchased from TRC (Toronto Research Chemicals, Ontario, Canada). The internal standard was 4-methyl-mexirethyn.

Pharmacokinetic sampling and assay. Blood sampling (pre-dose, and 2, 4, 6, 8, 10 and $24 \mathrm{~h}$ post-dose) was performed on day 7 of course 2 (sunitinib, $25 \mathrm{mg}$ ). Samples $(0.5 \mathrm{ml})$ were collected in tubes containing ethylenediamine tetraacetic acid (EDTA). Samples were centrifuged at $3,500 \mathrm{rpm}$ at $4^{\circ} \mathrm{C}$ for 10 min. $\mathrm{NaOH}(0.1 \mathrm{~N})$ was added to the supernatants, and the compounds were extracted into $3 \mathrm{ml} t$-butyl methyl ether (TBME) and agitated for $5 \mathrm{~min}$. The TBME phase was aspirated and evaporated to dryness $\left(\mathrm{N}_{2}\right)$. Aliquots were subjected to high-performance liquid chromatography. The protocol

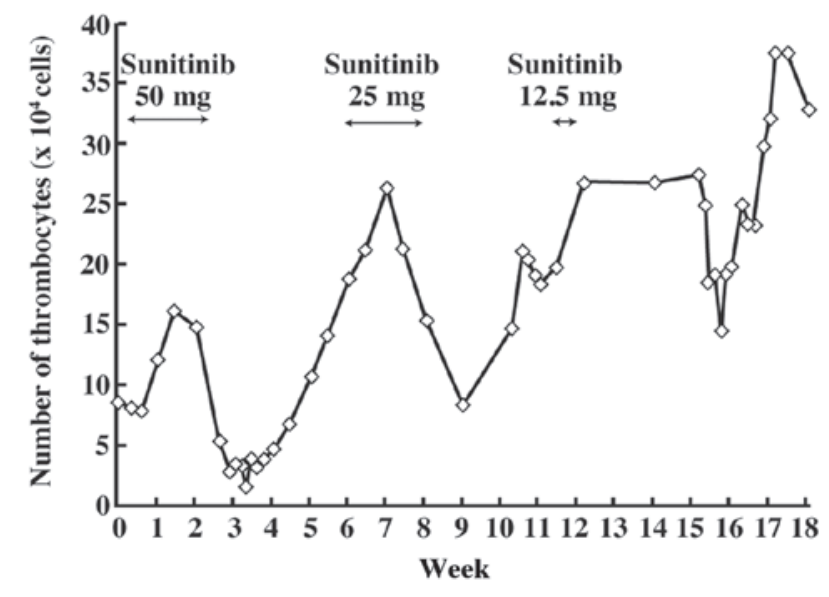

Figure 1. Thrombocytopenia following administration of sunitinib.

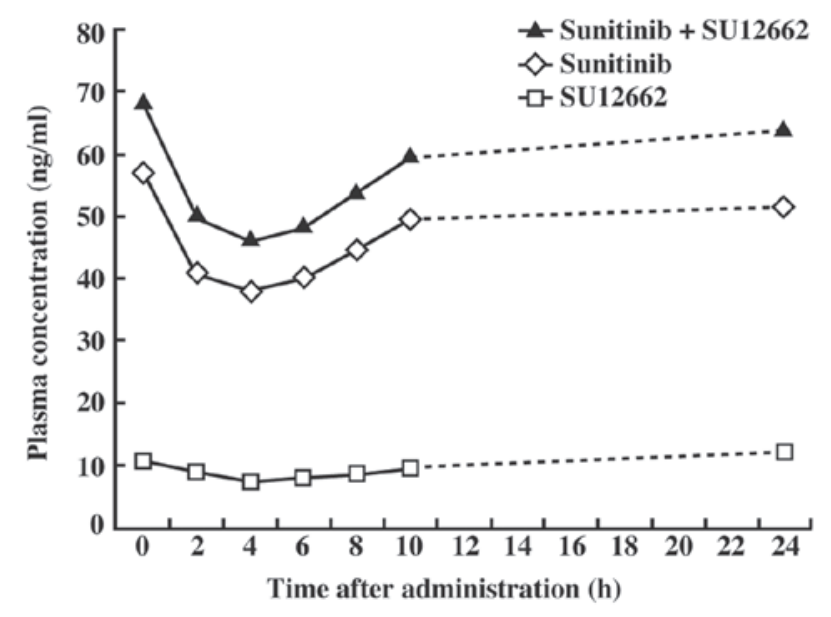

Figure 2. Diurnal variation of plasma concentration 7 days after starting sunitinib administration ( $25 \mathrm{mg} /$ day $)$.

was approved by the Medical Ethics Committee of Shinshu University and the patient provided informed consent prior to the study.

High-performance liquid chromatography conditions. The chromatographic system consisted of a mobile phase of mixture [0.05 $\mathrm{M}$ phosphoric buffer ( $\mathrm{pH} 3$ ), acetonitrile and B-7 low UV reagent (Waters, Milford, MA, USA) at a ratio of 695:300:5] with an ODS column pumped at a flow rate of $0.3 \mathrm{ml} / \mathrm{min}$ and UV/VIS detection at $431 \mathrm{~nm}(0-12 \mathrm{~min})$ and $250 \mathrm{~nm}(12-20 \mathrm{~min})$ (7). The retention times for $N$-desethyl sunitinib, sunitinib and internal control were 5.8, 8.3 and 14.8 min, respectively.

\section{Results}

The trough concentrations of sunitinib and SU12662 in plasma after 7 days at $25 \mathrm{mg}$ were 38.0 and $7.4 \mathrm{ng} / \mathrm{ml}$, respectively, and that of sunitinib plus SU12662 was $46.1 \mathrm{ng} / \mathrm{ml}$. The average concentration was $56.0 \mathrm{ng} / \mathrm{ml}$ (Fig. 2). The area under the curve (AUC) of sunitinib plus SU12662 was 1,393.0 ng.h/l. 


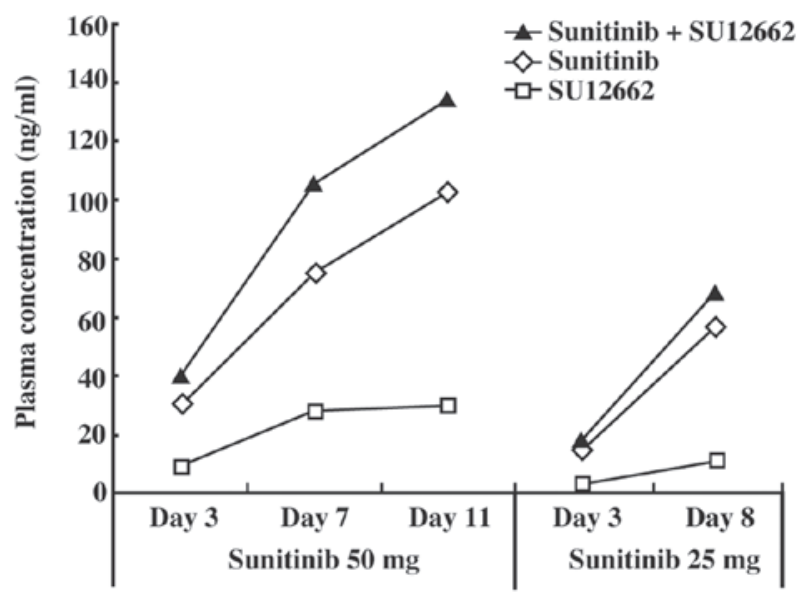

Figure 3. Progress of plasma concentration from the start of sunitinib administration $(50 \mathrm{mg} /$ day or $25 \mathrm{mg} /$ day).

The concentrations of sunitinib, SU12662, and sunitinib plus SU12662 in plasma at $50 \mathrm{mg}$ were $30.2,9.2$ and $40.1 \mathrm{ng} / \mathrm{ml}$ (3 days); 75.2, 28 and $105.3 \mathrm{ng} / \mathrm{ml}$ (7 days); and 102.3, 29.5 and $134.0 \mathrm{ng} / \mathrm{ml}$ (14 days), respectively (Fig. 3).

\section{Discussion}

A plasma concentration above $50 \mathrm{ng} / \mathrm{ml}$ of sunitinib plus SU12662 is required for the inhibition of tyrosine kinase phosphorylation. Fig. 3 shows that the plasma concentrations before administration of sunitinib and sunitinib plus SU12662 11 days after initiation of sunitinib treatment $(50 \mathrm{mg} /$ day) were approximately 100 and $120 \mathrm{ng} / \mathrm{ml}$, respectively. These concentrations were not troughs since the trough concentration did not correlate with the plasma concentration prior to the administration of sunitinib, as shown in Fig. 2. The results shown in Fig. 2 indicated that the trough concentration of sunitinib (50 mg) plus SU12662 at 11 days would likely be over $50 \mathrm{ng} / \mathrm{ml}$. Sunitinib inhibits Flk-1/KDR and PDGFR phosphorylation at concentrations over the range of 50-100 ng/ml (sunitinib plus SU12662) in vivo. It has been reported that plasma concentrations of sunitinib and SU12662 should be maintained within the range of $50-100 \mathrm{ng} / \mathrm{ml}$ (4). The results shown in Fig. 3 indicate that the plasma concentration of sunitinib was sufficient to inhibit Flk-1/KDR and PDGFR in this patient.

Pharmacokinetics in patients with GIST may be different from those in non-GIST patients. Notably, the concentration at $4 \mathrm{~h}$ after the administration of sunitinib was Cmin in Fig. 2. This patient underwent excision of the small intestine, and it was considered that this excision delayed the absorption of sunitinib. Many patients with small intestinal GIST undergo excision of the small intestine, and it is therefore predicted that sunitinib absorption will be delayed in such cases. Furthermore, the plasma concentrations of SU12662 on days 7 and 14 at $50 \mathrm{mg}$ sunitinib were the same. This result was due to the reduced CYP3A4 activity caused by the tumor metastasis in the left side of the liver.

Reduction of sunitinib may not suppress thrombocytopenia at the effective concentration. Recently, it was reported that the adverse effects of sunitinib are correlated with plasma concentration (8). Diastolic blood pressure has been shown to be correlated with trough plasma concentration of total drug (sunitinib plus SU12662). Conversely, the absolute neutrophil count is correlated with AUCcum28tot (28-day cumulative AUC of total drug) (8). However, in this patient, the reduction in plasma concentration did not ameliorate thrombocytopenia.

This case report suggests that sunitinib at a dose of $50 \mathrm{mg}$ could be an over-dosage in Asian women. Houk et al reported that the AUC and Cmax of both sunitinib and total drug are increased in Asians and in females (9). This was suggested to be associated with a reduction in CYP3A4 activity since both sunitinib and SU12662 are metabolized only by CYP3A4. Metabolism in the liver is associated with liver volume (10). If the liver volume is small, the metabolic capability is also small. The liver volume is small in Asians and females since the average weight and height are generally lower than those of non-Asians and males. Therefore, it is possible to calculate liver volume by body surface area (11). The results suggested that the metabolic capability of CYP3A4 is lower in Asian than non-Asian females.

CYP3A5 is also likely to demonstrate differences in metabolic ability among individuals. It is difficult to separate the effects of CYP3A5 and CYP3A4 as they have similar spectra of substrates. CYP3A5 is the predominant isozyme among human liver CYP3As (12), and CYP3A5 may be responsible for the metabolism of sunitinib. However, it has been reported that there are ethnic and individual differences in the expression of CYP3A5. The activity of CYP3A5.3 is extremely low and this $C Y P 3 A 5^{*} 3$ genotype was $60 \%$ in Japanese or Chinese, $31 \%$ in Indian, $70 \%$ in Caucasians, and $35 \%$ in African-Americans (13-15). Thus, it is likely to be involved in the differences in expression of hepatic CYP3A between ethnicities and individuals. These differences suggested that plasma concentration of sunitinib differs between individuals.

We concluded that the monitoring of sunitinib plasma concentration is essential to determine the appropriate dose in individual patients. Furthermore, this study showed that the frequency of thrombocytopenia and hypothyroidism in Asians, including Japanese, with sunitinib is higher than in Europeans and Americans. This is because the plasma concentrations in Asians are higher than those in Europeans and Americans due to the liver volume being smaller and due to $C Y P 3 A 5^{*} 3$ being the major genotype in Asians.

\section{Acknowledgements}

We thank Dr Hajime Ichimura for providing clinical insights and taking blood samples.

\section{References}

1. Rubin BP: Gastrointestinal stromal tumours: an update. Histopathology 48: 83-96, 2006.

2. Hormick JL and Fletcher CD: The role of KIT in the management of patients with gastrointestinal stromal tumours. Hum Pathol 38: 679-687, 2007.

3. Debiec-Rychter M, Sciot R, Le Cesne A, et al: KIT mutations and dose selection for imatinib in patients with advanced gastrointestinal stromal tumours. Eur J Cancer 42: 1093-1103, 2006.

4. Mendel DB, Laird AD, Xin X, et al: In vivo antitumour activity of SU11248, a novel tyrosine kinase inhibitor targeting vascular endothelial growth factor and platelet-derived growth factor receptors: Determination of a pharmacokinetic/pharmacodynamic relationship. Clin Cancer Res 9: 327-337, 2003. 
5. Murray LJ, Abrams TJ, Long KR, et al: SU11248 inhibits tumor growth and CSF-1R-dependent osteolysis in an experimental breast cancer bone metastasis model. Clin Exp Metastasis 20: 757-766, 2003

6. Chen M, Nafziger AN and Bertino JS Jr: Drug-metabolizing enzyme inhibition by ketoconazole does not reduce interindividual variability of CYP3A activity as measured by ora midazolam. Drug Metab Dispos 34: 2079-2082, 2006.

7. Blanchet B, Saboureau C, Benichou AS, et al: Development and validation of an HPLC-UV-visible method for sunitinib quantification in human plasma. Clin Chim Acta 404: 134-139, 2009.

8. Houk BE, Bello CL, Poland B, Rosen LS, Demetri GD and Moltzer RJ: Relationship between exposure to sunitinib and efficacy and tolerability endpoints in patients with cancer: results of a pharmacokinetic/ pharmacodynamic meta-analysis. Cancer Chemther Pharmacol 66: 357-371, 2010.

9. Houk BE, Bello CL, Kang D and Amantea M: A population pharmacokinetic meta-analysis of sunitinib malate (SU11248) and its primary metabolite (SU12662) in healthy volunteers and oncology patients. Clin Cancer Res 15: 2497-2506, 2009.
10. Nakazawa Y, Chisuwa H, Ikegami T, et al: Relationship between in vivo FK506 clearance and in vitro 13-demethylation activity in liver-related liver transplantation. Transplantation 66: 1089-1093, 1998.

11. Urata K, Kawasaki S, Matsunami H, et al: Calculation of child and adult standard liver volume for liver transplantation. Hepatology 21: 1317-1321, 1995.

12. Kuehl P, Zhang J, Lin Y, et al: Sequence diversity in $C Y P 3 A$ promoters and characterization of the genetics basis of polymorphic CYP3A5 expression. Nat Genet 27: 383-391, 2001.

13. Saeki M, Saito Y, Nakamura T, et al: Single nucleotide polymorphisms and haplotype frequencies of CYP3A5 in a Japanese population. Hum Mutat 21: 653, 2003.

14. Balram C, Zhou Q, Cheung YB and Lee EJ: CYP3A5*3 and *6 single nucleotide polymorphisms in three distinct Asian populations. Eur J Clin Pharmacol 59: 123-126, 2003.

15. Fukuen S, Fukuda T, Maune H, et al: Novel detection assay by PCR-RFLP and frequency of the CYP3A5 SNPs, CYP3A5*3 and $* 6$, in a Japanese population. Pharmacogenetics 12: 331-334, 2002 . 